\title{
Ribosomal Interaction
}

National Cancer Institute

\section{Source}

National Cancer Institute. Ribosomal Interaction. NCI Thesaurus. Code C40539.

Ribosomal Interaction involves temporary non-covalent binding between biomolecules and ribosomes. 